\title{
Coronal Mass Ejection, Magnetic Cloud and their Geoeffectiveness During 1996-2009
}

\author{
Rohit Verma ${ }^{1}$, Santosh Kumar ${ }^{2}$, Shushil Kumar Dubey ${ }^{3}$ \\ ${ }^{1}$ S.G.T.B.Khalsa College, Jabalpur (M.P.) - 482001. India \\ ${ }^{2}$ H.No.992/3036, Jabali Dant Chikitsalaya, Survey of India, Behind SBI Qrts., Vijay Nagar, Jabalpur (M.P.) - 482001. India \\ ${ }^{3}$ Auto. Govt. Science College, Jabalpur (M.P.) - 482001. India
}

\begin{abstract}
A detailed investigation on geoeffectiveness of Coronal Mass Ejections [ CMEs ] associated with Magnetic Clouds [ MCs ] observed during 1996-2009 have been studied. The collected sample events are divided into two groups based on their association with CMEs related to geomagnetic storms Dst $\leq-50 \mathrm{nT}$ eg. 1. geoeffective events and 2. For nongeoeffective events Dst $\geq-50 n T$. Furthermore, most of the CMEs are of halo type occurred mostly in western hemisphere. Halo and partial halo CMEs are likely to be the major cause for Geomagnetic storms [GMSs ] of high intensity. There exists a weak anti-correlationship $(R=-0.36)$ for geoeffective events between $C M E$ speed $\left[V_{C M E}\right]$ and Dst index and relatively better correlation 1. between $V_{C M E}$ and solar wind speed [ $\left.V_{S W}\right](R=0.54)$ and $2 . D s t$ index and solar wind speed ( $R=0.64)$; whereas, the correlation $(R=0.16)$ between Dst index and southward magnetic field component I $\left.B_{Z}\right]$ is very poor. From our findings, we have observed that the intense and long duration, southward magnetic field component $\left[B_{Z}\right]$ and fast solar wind speed are responsible for geomagnetic storms, and geomagnetic storms weakly depend on CME velocity.
\end{abstract}

Keywords: Coronal Mass Ejections; Magnetic Cloud; Solar Wind ; Geomagnetic Stroms

PACS Nos. 96.60.ph ;96.50.Uv ;96.50.Ci

\section{Introduction}

In recent years a number of investigations have been carried out to understand the solar terrestrial relationship and to ascertain factors that are responsible for GMSs, Gopalswamy $\mathrm{N}$, Akiyama S, Yashiro S, Michalek $\mathrm{G}$ and Lepping R P,2008 and Kumar S and Raizada A 2008. It is believed that the GMSs are the response to interplanetary [ IP ] phenomena arising as a consequence of a solar event. The geospheric environment is highly affected by the Sun and its features such as Solar Flares [ SFs ], Active Prominences and Disappearing Filaments [ APDFs ], Coronal Holes [ CHs ], CMEs etc. Research since last three decades identifies CMEs as the energetic events in the heliosphere. CMEs from the Sun derive, Solar Wind (SW) disturbances in terms of magnetic field, speed and density which in turn causes geomagnetic disturbances at Earth.

Due to the effects of Solar flare and CME events, a GMS of longer than average duration may result. The intensity of GMSs is primarily decided by CMEs speed and strength of magnetic field it contains, Gopalswamy N 2006 and Cane H V, Richardson I G and St Cyr O C 2000; whereas, according to Manoharan P K 2006 primary factors determining the geoeffectiveness are : the direction of propagation of CMEs, its speed, size, density and further, orientation and strength of the magnetic field at the near Earth space. Intense GMSs are found to be mainly caused by CMEs by Zhang J, Dere K P, Howard R A and Bothmer V, 1996. The frequency of CMEs vary with sunspot cycle.

CMEs are large scale plasma and magnetic field structures moving away from the Sun into heliosphere (Gopalswamy $\mathrm{N}, 2002$ ) . CMEs, which appear to surround the occulting disk of the observing coronagraph are known as halo CMEs (Howard R A, Michels D J, Sheeley N R and Koomen M J,
1982). The CMEs with an apparent width of $360^{\circ}$ are taken as halo; whereas, the CMEs with width $\leq 359^{\circ}$ and $120^{\circ}$ are taken as ,partial halo"e (Loewe C A and Prolss G W, 1997 and Gopalswamy N, Yashiro S and Akiyama S, 2007) . CMEs that are observed in the solar wind near 1 AU are commonly called interplanetary coronal mass ejection [ICMEs]. MC is the subset of ICMEs having a specific configuration in which the magnetic strength is higher than the average magnetic field. Halo CMEs have now been shown to be an important factor affecting the physical conditions in the entire Heliosphere. Ability of CMEs to cause GMSs is known as geoeffectiveness which is measured in the terms of geomagnetic index such as the disturbance storm time of Dst. A MC is a transient event observed in the solar wind. It is defined as a region of enhanced magnetic field strength. smooth rotation of the magnetic field vector and low proton temperature(Burlaga L F, Sittler E, Mariani F and Schwenn R, 1981). MCs are a possible manifestation of CMEs.

\section{Data Analysis}

During the period 1996 -2009 CMEs associated with magnetic clouds have been investigated. The data on Magnetic Clouds is obtained from MFI table of MAG CLOUD'S ( File://G: MMFI TABLE OF MAG CLOUD'S.htm ) covering the above period and containing 110 events. The values of Dst indices are taken from world data center, Japan ( http://swdcwww.kugi.kyoto-u.ac.jp). The data regarding the related CMEs (speed, width and acceleration) are taken from the online $\mathrm{SOHO} / \mathrm{LASCO} \mathrm{CME}$ catalog maintained by the CDAW data center ( http://cdaw.gsfc. nasa.gov/CME list ). The data on Shocks is obtained from ACE Lists of Disturbances and Transients. Out of 110 events only 101 events are taken for our further analysis using the following selection criteria 1 . The given CMEs information should be 


\section{International Journal of Science and Research (IJSR) \\ ISSN (Online): 2319-7064 \\ Index Copernicus Value (2013): 6.14 | Impact Factor (2015): 6.391}

Clear 2. The days with data being not available are excluded from the analysis.

The above two selection criteria are used to select the clear events from which the properties of CMEs and their associated Magnetic Clouds can be studied clearly. The events are considered to be geoeffective if their Dst index $\leq-$ $50 \mathrm{nT}$, (Gopalswamy N, Yashiro S and Akiyama S, 2007 ) 5 . The sample events are divided into two groups as follows; 1. Geoeffective events : It contains 54 events corresponding Dst index values $\leq-50 \mathrm{nT} 2$. Nongeoeffective events : It contains the remaining 47 events.

\section{Results and Discussion}

The CME speed listed in the LASCO CME catalog is measured from the height time measurements projected in the sky plane. So all the measured parameters will suffer from projection effects. No attempt has been made to correct the projection effects. The speed of the CMEs varies from few hundred $\mathrm{Km} / \mathrm{s}$ to $2000 \mathrm{Km} / \mathrm{s}$. The minimum and maximum value of CME velocity is observed to be 104 and $2115 \mathrm{Km} / \mathrm{s}$, respectively nongeoeffective events; whereas, for geoeffective events the minimum and maximum values are 108 and $2465 \mathrm{Km} / \mathrm{s}$, respectively. The mean speed of nongeoeffective events is $468.51 \mathrm{Km} / \mathrm{s}$ and that of the geoeffective events is $787 \mathrm{Km} / \mathrm{s}$. The weak dependence between Vcme and the Dst indicates that the initial speed of CME might not be used as the parameter for predicting geomagnetic activity, which is also observed by Zhang J, Dere K P, Howard R A and Bothmer V, $2003^{11}$.

The minimum and maximum values of SW velocity are observed to be 275 and $640 \mathrm{Km} / \mathrm{s}$, respectively nongeoeffective events; whereas, for geoeffective events the minimum and maximum values are 315 and $990 \mathrm{Km} / \mathrm{s}$, respectively. The mean speed of nongeoeffective events is $396.8 \mathrm{Km} / \mathrm{s}$ and that of the geoeffective events is 508.16

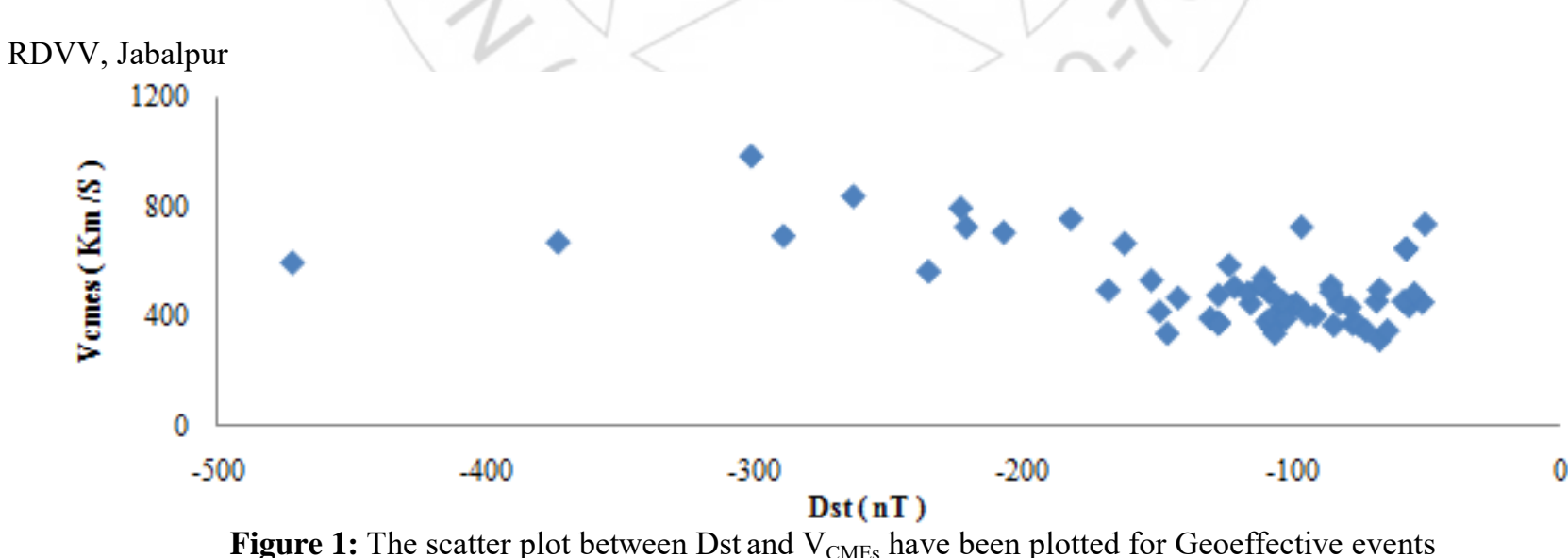

Volume 5 Issue 5, May 2016 www.ijsr.net 
RDVV, Jabalpur

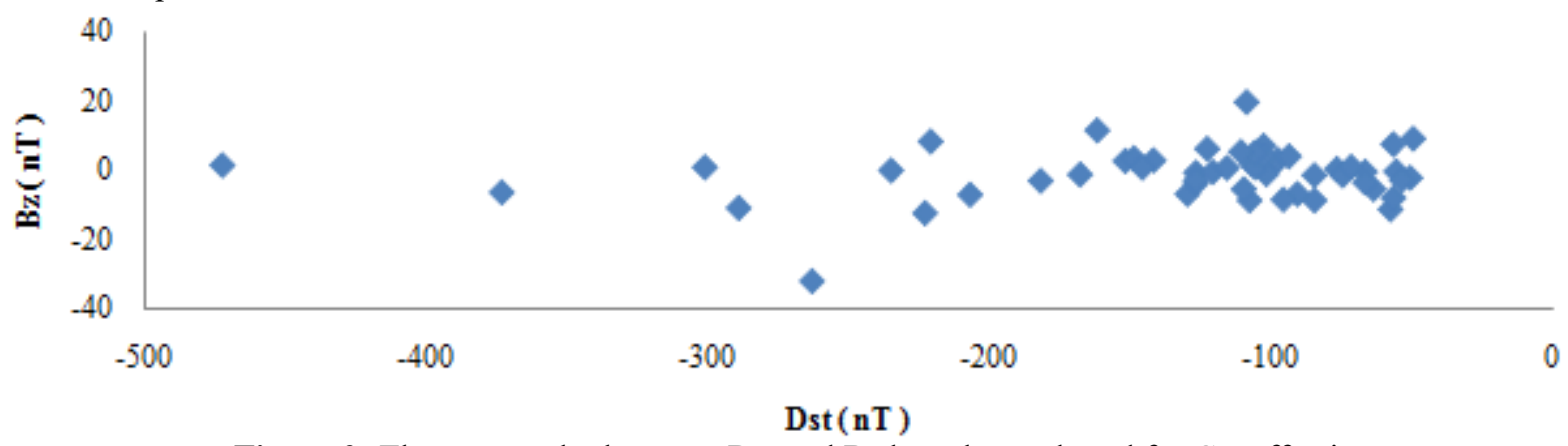

Figure 2: The scatter plot between Dst and $B_{Z}$ have been plotted for Geoeffective events

RDVV, Jabalpur

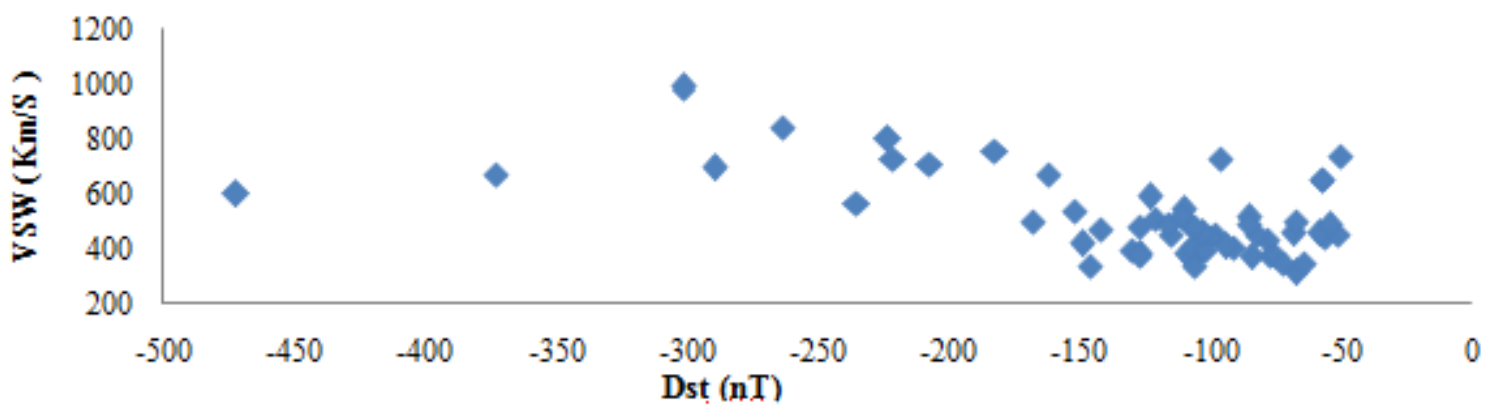

Figure 3: The scatter plot between Dst and $\mathrm{V}_{\mathrm{SW}}$ shave been plotted for Geoeffective events

RDVV, Jabalpur

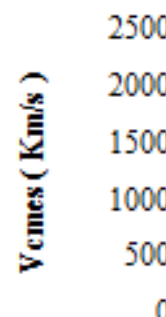

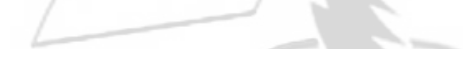

200

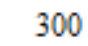

$\mathrm{sw}(\mathrm{Km} / \mathrm{s})$

Figure 4: The scatter plot between $V_{\mathrm{SW}}$ and $\mathrm{V}_{\mathrm{CMEs}}$ have been plotted for Geoeffective events RDVV, Jabalpur

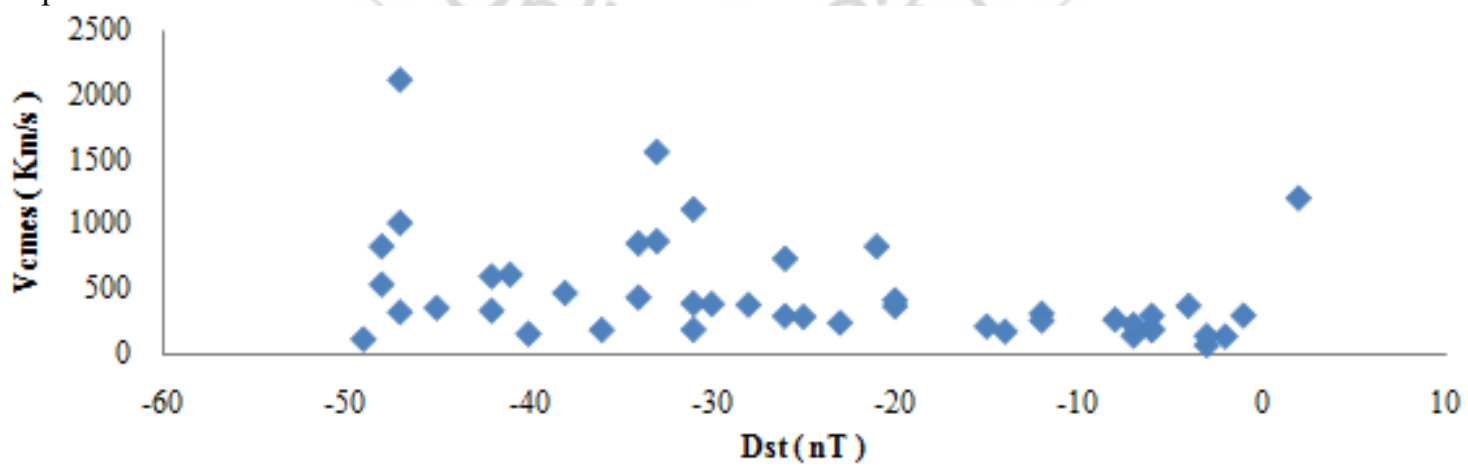

Figure 5: The scatter plot between Dst and $\mathrm{V}_{\mathrm{CMEs}}$ have been plotted for Nongeoeffective events 


\section{International Journal of Science and Research (IJSR) \\ ISSN (Online): 2319-7064}

Index Copernicus Value (2013): 6.14 | Impact Factor (2015): 6.391

RDVV, Jabalpur

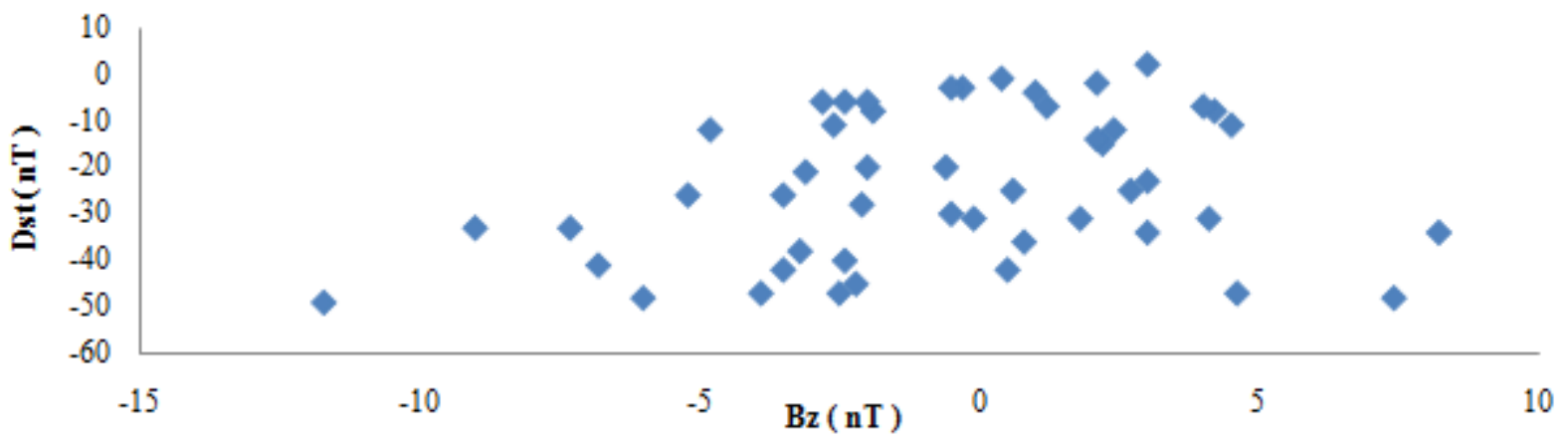

Figure 6: The scatter plot between $\mathrm{B}_{\mathrm{Z}}$ and Dst have been plotted for Nongeoeffective events

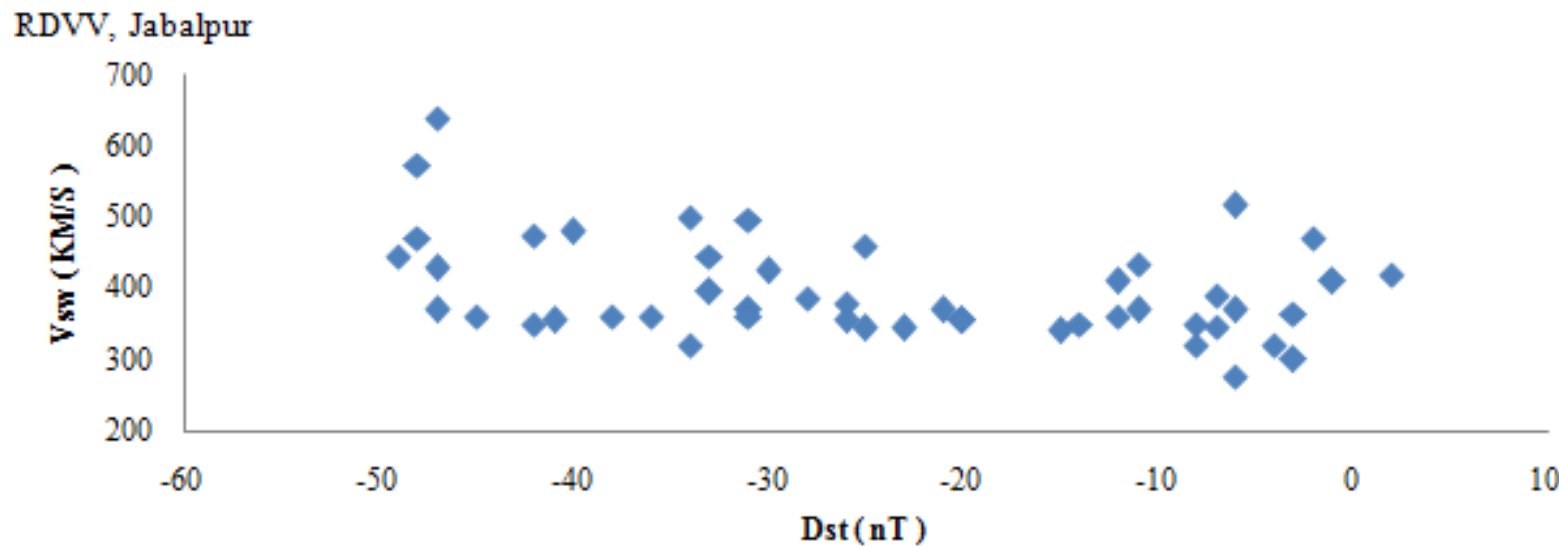

Figure 7: The scatter plot between Dst and $V_{\text {SW }}$ have been plotted for Nongeoeffective events

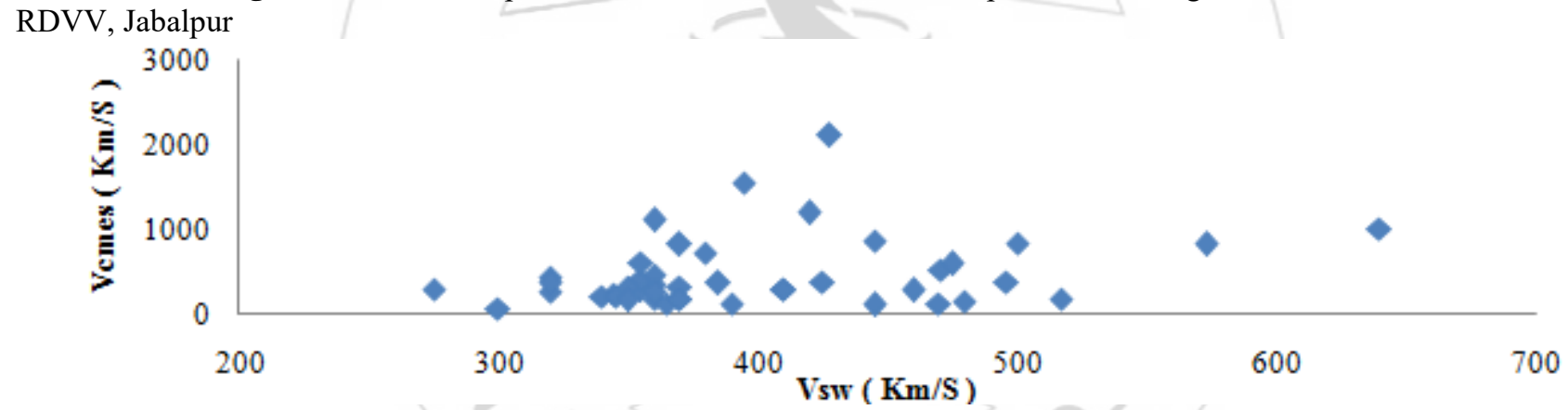

Figure 8: The scatter plot between $\mathrm{V}_{\mathrm{SW}}$ and $\mathrm{V}_{\mathrm{CMEs}}$ have been plotted for Nongeoeffective events

There exists a weak anti-correlation between CME speed and Dst index for nongeoeffective events with an anti-correlation coefficient ( $\mathrm{R}=-0.37$ ) as shown in Fig. - 5. Further there exists a weak correlation ( $\mathrm{R}=0.24$ ) between Dst index and southward magnetic field component $\left[\mathrm{B}_{\mathrm{Z}}\right]$ for geoeffective events as shown in Fig. - 6.

The anti-correlation between solar wind speed and Dst index for nongeoeffective events are shown in Fig. -7. There exists a good correlation between Dst index and solar wind speed $(\mathrm{R}=-0.38)$. There exists a weak correlation $(\mathrm{R}=0.29)$ between $\mathrm{CME}$ speed and solar wind speed for nongeoeffective events as shown in Fig. -8 .

Speed of MCs seem to be one of the important parameters responsible for the occurrence of GMSs. There exists a good anti-correlation $(\mathrm{R}=-0.64)$ between magnetic cloud velocity and Dst index. Geoeffective MCs generally have high speed. Speed of MCs appear to have greater impact on the minimum value of Dst attained during a GMSs. Furthermore, it has been observed that the MCs are about $47 \% \mathrm{RH}$ and $53 \%$ LH that have occurred.

Furthermore, the effect of CMEs (i.e.; halo as well as partial halo) to cause GMSs of varying nature is investigated. It is found that $53 \%$ halo and $47 \%$ partial halo CMEs are responsible for GMSs. Thus, it may be deduced that CMEs having Position Angle [PA] greater or equal to $120^{\circ}$ have higher probability of reaching the Earth than other CMEs of having PA less than $120^{\circ}$.

It is observable from here that MCs having a velocity greater than $400 \mathrm{Km} / \mathrm{s}$ are mostly responsible for GMSs. Thus, it is deduced that MCs velocity also play an important role in the prediction of GMSs.

\section{Conclusions}

101 CMEs associated with MCs observed during 1996 2009 are analyzed. The sample events are divided into two groups based on the CMEs association with Dst $\leq-50 \mathrm{nT}$ as 


\section{International Journal of Science and Research (IJSR) \\ ISSN (Online): 2319-7064 \\ Index Copernicus Value (2013): 6.14 | Impact Factor (2015): 6.391}

(i)geoeffective events and (ii) nongeoeffective events . The results of our study are summarized as follow: It may be deduced that CMEs having Position Angle [ PA ] greater or equal to $120^{\circ}$ have produced geomagnetic storms and CMEs velocity is also a good predictor of geomagnetic storms. The velocity of CMEs has been found to play major role in deciding the strength of GMSs alongwith other parameters like angular width, direction of motion etc. It is expected that ones with more speed leads to more disturbance in magnetosphere, which in turn causes intense GMSs. The CMEs with less speed leads to weak GMSs. Most of the geoeffective CMEs have occurred in western hemisphere.

The magnetic clouds having velocity greater than $400 \mathrm{~km} / \mathrm{s}$ are mostly responsible for GMSs. Magnetic cloud velocity has significant correlation with Dst and it is also one of the important parameter for prediction of the GMSs.

The anti-correlation between CME speed and Dst is weak for geoeffective events $(\mathrm{R}=-0.24)$ and weak for nongeoeffective event $(\mathrm{R}=-0.37)$. The anti-correlation between Dst index and solar wind speed is good for geoeffective events $(\mathrm{R}=-0.56)$ and weak anti-correlation for nongeoeffective events $(\mathrm{R}=-0.38)$. The correlation between Dst index and southward magnetic field component [Bz] is weak for geoeffective events $(\mathrm{R}=0.21)$ and for nongeoeffective event $(\mathrm{R}=0.24)$. The correlation between CME speed and solar wind speed is good for geoeffective events $(\mathrm{R}=0.54) \&$ is weak for nongeoeffective events ( $\mathrm{R}$ $=0.29)$.

The high speed solar wind plasma may be in the form of CMEs or else, is more likely to cause the intense and super intense GMSs.

The geoeffective events are found to be associated with intense geomagnetic storm with mean Dst index (-128.55 $\mathrm{nT}$ ). The nongeoeffective events are found to be associated with weak geomagnetic storm with mean Dst index (-23.979 nT). Furthermore, the effect of CMEs ( i.e; halo as well as partial halo ). There exists a weak correlation between (i) CME speed and Dst index with an anti-correlation coefficient of $\mathrm{R}=-0.34$ (ii) Dst index and southward magnetic field component $[\mathrm{Bz}]$ with a anti-correlation coefficient of $\mathrm{R}=-0.4$. There exists a good anti-correlation between (i) Dst index and solar wind speed with a anticorrelation coefficient of $\mathrm{R}=-0.64$.

\section{Acknowledgements}

The authors are highly indebted to various experimental groups for providing data online through Internet. They are also grateful to Prof. N. Gopalswamy and other authors for providing valuable information on CMEs directly or indirectly. The authors acknowledge the contributions from several of his colleagues.

\section{References}

[1] Burlaga L F, Sittler E, Mariani F and Schwenn R "Magnetic loop behind an interplanetary shock" Journal of Geophysical Research Vol.86 (1981) 6673.
[2] Cane H V, Richardson I G and St Cyr O C "Coronal mass ejections,interplanetary ejecta and geomagnetic storms" Geophys. Res. Lett. 27 (2000) 3591.

[3] Gopalswamy N "Relation between CMEs and ICMEs" COSPAR Colloquia Series edited by $H N$ Wang and $R L$ $X u$ Vol.14 (2002) 157 (and references therein).

[4] Gopalswamy N "Coronal mass ejections of solar cycle 23" J. Astrophys. Astronomy Vol.27 ( 2006) 243 (and references therein).

[5] Gopalswamy N, Yashiro S and Akiyama S "Geoeffectiveness of halo coronal mass ejections" Journal of Geophysical Research Vol.112 (2007) A06112.

[6] Gopalswamy N, Akiyama S, Yashiro S, Michalek G and Lepping R P " Solar sources and geospace consequences of interplanetary magnetic clouds observed during solar cycle 23" J. Atmos. Sol. Terr. Phys. Vol. 70 (2008) 245.

[7] Howard R A, Michels D J, Sheeley N R and Koomen M $\mathrm{J}$ "The observation a coronal transient directed at earth" The astrophysical journal letter 263 (1982) L101-L104 .

[8] Kumar S and Raizada A "Effect of solar features and interplanetary parameters on geomagnetosphere during solar cycle-23" Pramana Journal of Physics Vol.71, 6 (2008) 1353.

[9] Loewe C A and Prolss G W "Classification and mean behavior of magnetic storms" Journal of Geophysical Research Vol.102 (1997) 14,209.

[10] Manoharan P K "Evolution of Coronal Mass Ejections in the Inner Heliosphere: A Study Using White-Light and Scintillation Images" Solar physics Vol.235 (2006) 345.

[11]Zhang J, Dere K P, Howard R A and Bothmer V "Identification of solar sources of major geomagnetic storms between 1996 and 2000" Astrophys J. Vol.582 (2003) 520 (and references therein) .

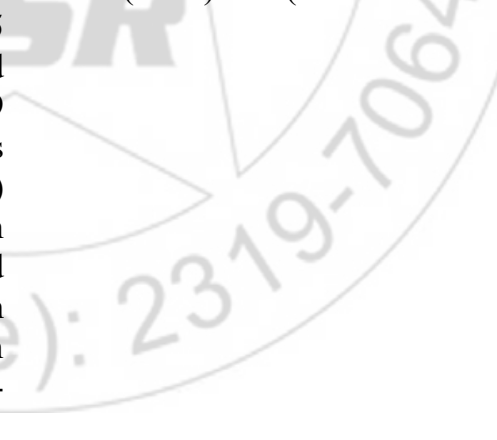

